\title{
Adam Smith y la Belleza de la Ciencia
}

\author{
Jorge López Lloret ${ }^{1}$
}

Recibido: 03 de abril de 2017 / Aceptado: 29 de enero de 2018

Resumen. El presente artículo analiza la concepción de la metodología científica expresada por Adam Smith en su historia de la astronomía, algo importante para comprender el resto de su obra. Los estudiosos de este tema se han centrado en su mayor parte en la influencia de Newton y Hume sobre Smith, surgiendo una concepción en la que la dimensión epistemológica y la estética se relacionan de una manera tensa. El autor identifica nuevas fuentes, de naturaleza estética, que se integran en este debate y ayudan a aclararlo: Addison, Crousaz, Hutcheson, Berkeley y Diderot, cuya obra Smith conoció y apreció. A partir de ahí llega a la conclusión de que Smith puso en práctica una metodología rica y compleja en la que los elementos epistemológicos y culturales se relacionaron de una manera no tensa ni excluyente, sino armónica e incluyente.

Palabras clave: Adam Smith, belleza; estética; metodología científica; Newton; siglo XVIII.

\section{[en] Adam Smith and the Beauty of Science}

Abstract. This article analyses the conception of the scientific methodology expressed by Adam Smith in his history of astronomy, something important to understand the rest of his work. The current state of the studies on this subject has focused largely on Newton and Hume influence on Smith, arising a conception in which the epistemological dimension and the aesthetics are related in a controversial manner. The author identifies new aesthetic sources that are integrated in this debate and help to clarify it: Addison, Crousaz, Hutcheson, Berkeley and Diderot, whose work Smith knew and appreciated. From there he concludes that Smith put into practice a rich and complex methodology in which the epistemological and cultural elements were related in one way not controversial nor excluding, but harmonious and inclusive.

Keywords: Adam Smith; beauty; aesthetics; scientific methodology; Newton; eighteenth century.

Sumario: 1. Introducción; 2. La Historia de la Astronomía y la fórmula de la belleza; 3. La transversalidad de la máquina; 4. La fuerza constructiva de la imaginación; 5. Conclusiones; 6. Referencias bibliográficas.

Cómo citar: López Lloret, J. (2019): “Adam Smith y la Belleza de la Ciencia”, en Revista Anales del Seminario de Historia de la Filosofia 36 (1), 87-106.

$1 \quad$ Universidad de Sevilla

lopezlloret@us.es

ORCID: 0000-0002-6738-5895 


\section{Introducción}

En 1803 decía John Millar de la filosofía social de Adam Smith lo siguiente:

The great Montesquieu pointed out the road. He was the Lord Bacon in this branch of Philosophy. Dr. Smith is the Newton. ${ }^{2}$

Esta comparación de Adam Smith con Isaac Newton se puede interpretar como algo muy concreto o muy genérico. Seguramente Millar lo vio de la segunda manera, pues Newton en ese momento personalizaba el éxito científico y, como el mismo Smith advirtió, la importancia de un "filósofo" se realzaba calificándolo de Newton. ${ }^{3}$

Esto no hubiera pasado de ser una metáfora genérica si no fuera porque Smith escribió una historia de la astronomía que llegaba hasta Newton, ${ }^{4}$ basándose en la cual los analistas de su obra han desarrollado una investigación minuciosa del influjo de Newton sobre Smith, cuyo interés por él fue común a toda la Ilustración Escocesa. ${ }^{5}$ Entre las muchas interpretaciones posibles de dicho influjo, ${ }^{6}$ nosotros nos alineamos con los que defienden su naturaleza metodológica, anunciada por Newton al final de su Óptica:

\footnotetext{
Citado por Scott, W.R. Adam Smith as Student and Professor. Glasgow: Jackson, Son \& Company, 1937, 56.

Smith, Adam. Lectures on Rhetoric and Belles Lettres. Indianápolis: Liberty Fund, 1985, 204.

4 Se trata de The Principles which Lead and Direct Philosophical Enquiries; Illustrated by the History of Astronomy, en Smith, Adam. Essays on Philosophical Subjects. Indianápolis: Liberty Fund, 1992, 33-105 (hay traducción castellana de Carlos Rodríguez Braun: Ensayos filosóficos. Madrid: Pirámide, 1998, 43-112). En el cuerpo del artículo, a partir de ahora citaremos como HA; las citas serán de la edición castellana, aunque proporcionaremos la referencia de la inglesa. En las notas a pie de página nos referiremos a ésta como Astronomy y a aquélla como Astronomía. El texto se contextualiza en Ross, Ian Simpson. The Life of Adam Smith. Oxford: Clarendon Press, 1995, 97-108 y las introducciones a Astronomía (por John Reeder, pp. 28-32) y Astronomy (por W.P.D. Wightman, pp. 5-22).

5 Shepherd, Christine M. "Newtonianism in Scottish Universities in the Seventeenth Century", en R.H. Campbell y Andrew S. Skinner (eds.). The Origins and Nature of the Scottish Enlightenment. Edimburgo: John Donald Publishers, 1982, 65-85; WOOD, Paul. "Science in the Scottish Enlightenment", en Alexander Broadie (ed.). The Cambridge Companion to the Scottish Enlightenment. Cambridge: Cambridge University Press, 2010, 94-116 (especialmente 99-107); y Montes Lira, Leónidas. "Newtonianism and Adam Smith", en Christopher J. Berry; Maria Pia Paganelli; y Craig Smith (eds.). The Oxford Handbook of Adam Smith. Oxford: Oxford University Press, 2016, 36-53 (especialmente 42-50).

6 Influjo literal: Hetherington, Norriss S. "Isaac Newton's Influence on Adam Smith's Natural Laws in Economics". Journal of the History of Ideas, Vol. 44, No. 3, 1983, 497-505; Diemer, Arnaud y Guillemin, Hervé. "L'Économie Politique au Miroir de la Physique: Adam Smith et Isaac Newton". Revue d'Histoire des Sciences, 64, 2011, 5-26; Bréban, Laurie. "Smith oh Happiness: Towards a Gravitational Theory". European Journal of History of Economic Thought, Vol. 21, No. 3, 2014, 359-391.

Influjo metodológico: Montes Lira, Leónidas. "La Influencia de Newton en Adam Smith". Anuario Filosófico, XLII, 1, 2009, 137-158; Schliesser, Eric. "Copernican Revolutions Revisited in Adam Smith by Way of David Hume". Revista Empresa y Humanismo, Vol. XIII, 1, 2010, 213-248; Fiori, Stefano. "Adam Smith on Method: Newtonianism, History, Institutions, and the «Invisible Hand»". Journal of the History of Economic Thought, Vol. 34, 3, 2012, 411-435.

Influjo en ambos sentidos: Cremaschi, Sergio. "Adam Smith: Skeptical Newtonianism, Disenchanted Republicanism, and the Birth of Social Science", en Marcelo Dascal y Ora Gruengard (eds.). Knowledge and Politics. Boulder: Westview Press, 1989, 83-110; y Cremaschi, Sergio. "La Herencia Newtoniana en la Economía Política del Siglo XVIII", en Alberto Elena, Javier Ordóñez y Mariano Colubi (eds.). Después de Newton: ciencia y sociedad durante la primera Revolución Industrial. Barcelona: Anthropos, 1998, 70-101. Influjo metafórico: Kennedy, Gavin. "Adam Smith's Use of the «Gravitation» Metaphor". Economic Thought, $4,1,2015,67-79$.
} 
No sólo la filosofía natural se perfeccionará en todas sus partes siguiendo este método, sino que también la filosofía moral ensanchará sus fronteras. ${ }^{7}$

Smith fue influido por Newton a través de David Hume, quien adoptó el método experimental de razonamiento. Sin embargo, a diferencia de éste, que estableció un límite al conocimiento posible ${ }^{8}$ Smith aceptó la dinámica de la investigación científica que Newton había expuesto en la Óptica, en la que las fases de análisis y síntesis se sucedían generando un conocimiento cada vez más fiable de la realidad, ${ }^{9}$ aunque en el pensamiento de Newton había un poso de duda que se puede interpretar de una manera más o menos radical. Según la interpretación menos radical, pese a que nuestro conocimiento no accede a lo absoluto, no deja de aproximarse a ello. ${ }^{10}$ La más radical se basa en la completa falta de seguridad con respecto a que la naturaleza se adecue a las necesidades racionales de nuestro pensamiento. ${ }^{11}$ Debido a esto, como muchos de sus contemporáneos cuando se aproximaban a las cuestiones últimas, Newton recurrió a la garantía de Dios, aunque éste podría, si quisiera, variar las leyes de la naturaleza. ${ }^{12}$

La Teología Natural no fue, por lo tanto, injertada con violencia en la planta newtoniana, sino que brotaba de ella en sintonía con su medio ambiente, ${ }^{13}$ dado que dicha prueba de la existencia y naturaleza de Dios a partir del orden y belleza del mundo fue en general aceptada en Gran Bretaña en torno a los siglos XVII y XVIII. ${ }^{14}$ En ella se armonizaba la estructura del mundo que estaba descubriendo la nueva ciencia con su consistencia como obra de arte diseñada y producida por la mente divina. A comienzos del siglo XVIII, además, esto se tuvo que articular con la nueva subjetividad identificada por los empiristas británicos, dotada de una fuerte carga estética. ${ }^{15}$ Smith perteneció a este contexto, de manera que para comprender

7 Newton, Isaac. Óptica (1704). Traducción castellana de Carlos Solís. Madrid: Alfaguara, 1977, 350 (la edición inglesa consultada ha sido Opticks. Nueva York: Prometheus Books, 2003, 405). Smith recogió la idea en An Inquiry into the Nature and Causes of the Wealth of Nations (1776), Volume II. Indianápolis: Liberty Fund, 1981, 767-768 (citaremos como WN). La traducción castellana de Carlos Rodríguez Braun que hemos manejado ( $L a$ riqueza de las naciones. Madrid: Alianza, 1994 -citaremos como RN) sólo recoge una selección del Libro V, en la que no se incluye esta parte.

8 Hume, David. A Treatise of Human Nature (1739). Oxford: Clarendon Press, 1946, 13 (traducción castellana de Félix Duque: Tratado de la naturaleza humana. Madrid: Tecnos, 1988, 57). Véase Raphael, D.D. "«The true old Humean philosophy» and its influence on Adam Smith”, en G.P. Morice (ed.). David Hume. Bicentenary Papers. Edimburgo: Edinburgh University Press, 1977, 23-38.

9 Newton, Isaac. Principios matemáticos de la filosofía natural (1687). Traducción de Antonio Escohotado, Madrid, Tecnos, 1987, 6; Newton, Isaac. Opticks, 404 (Óptica, 349); Schliesser, Eric. "Copernican Revolutions Revisited in Adam Smith by Way of David Hume", 217-219; Montes Lira, Leónidas. "Newtonianism and Adam Smith", 46-50.

10 Newton, Isaac. Principios matemáticos de la filosofía natural, 463; NEWTON, Isaac. Opticks, 262, 370,404 (Óptica, 231, 320, 349).

11 Newton, Isaac. Four Letters from Sir Isaac Newton to Doctor Bentley (1756), en I. Bernard Cohen y Robert E. Schofield (eds.). Isaac Newton's Papers \& Letters on Natural Philosophy. Cambridge/Mass., Harvard University Press, 1978, 304. Véase la introducción a esta obra de Perry Miller. "Bentley and Newton", 271-278, especialmente 277 .

12 Newton, Isaac. Opticks, 403-404 (Óptica, 348-349). Véase Lázaro Cantero, Raquel. La sociedad comercial en Adam Smith. Método, moral, religión. Pamplona: EUNSA, 2002, 29-106.

13 Lázaro Cantero, Raquel. La sociedad comercial en Adam Smith, 49-78.

14 Jantzen, Benjamin C. An Introduction to Design Arguments. Cambridge: Cambridge University Press, 2014, 58-135.

15 Townsend, Dabney. "Lockean Aesthetics". The Journal of Aesthetics and Art Criticism, Vol. 49, No. 4, 1991, 349-361. 
su aproximación a la naturaleza de la ciencia debemos partir del mismo, incluso si aceptamos que, en connivencia con Hume, eliminó a la divinidad del escenario, pues no por ello tuvo que dejar de lado el empeño de aunar la estética y la ciencia, según el proyecto esbozado por su maestro Francis Hutcheson, a partir de la naturaleza humana. ${ }^{16}$

Nuestra intención es mostrar, centrándonos en HA, que Smith no sólo partió del newtonianismo al elaborar una teoría de la investigación científica como una actividad humana compleja que aunaba el conocimiento y el disfrute, la metodología y la cultura en igualdad de condiciones. Basta con que se consideren los libros de su biblioteca para comprender que su interés por ambas partes fue igualmente intenso, lo que hace llamativo que los analistas de este texto hayan dado más importancia a sus fuentes científicas. ${ }^{17}$ Pese a que varios estudiosos se han decantado por una interpretación estética de HA, se han centrado en su uso de la imaginación, proveniente de Hume, no interesándoles mucho la identificación de otras fuentes. ${ }^{18}$ Atendiendo a los contenidos temáticos de la obra e indagando en su biblioteca, a nosotros nos ha resultado clara la influencia de Joseph Addison, Jean-Pierre de Crousaz, Francis Hutcheson, George Berkeley y Denis Diderot, quienes resultan tan significativos como Newton, Maclaurin o Hume en la interpretación de su teoría de la ciencia y, a partir de ella, del estilo literario y las aportaciones científicas de su obra publicada, aunque esto último no lo estudiaremos en el presente artículo. ${ }^{19}$

En los próximos capítulos adoptaremos el siguiente plan: en primer lugar, mostraremos que la definición de ciencia proporcionada por Smith podría provenir tanto de Newton y Hume como de las propuestas estéticas de los autores enumerados. En segundo lugar, analizaremos la confluencia de la definición de la ciencia con la fórmula de la belleza en la noción de máquina, muy importante en el pensamiento de

16 Herman, Arthur. The Scottish Enlightenment. Londres: Fourth State, 2003, 73-74.

17 Hemos trabajado con los tres catálogos existentes de su biblioteca: Bonar, James. A Catalogue of the Library of Adam Smith. Londres: Macmillan and Co., 1932; Yanaihara, Tadao. Catalogue of Adam Smith's Library in the Possession of the University of Tokyo. Nueva York: Augustus M. Kelley, 1966; Mizuta, Hiroshi. Adam Smith's Library. A Catalogue. Oxford: Oxford University Press, 2000. Véase Smith, Craig. "The Essays on Philosophical Subjects", en Ryan Patrick Hanley (ed.). Adam Smith. His Life, Thought, and Legacy. Princeton: Princeton University Press, 2016, 89-104; Berry, Christopher J. "Adam Smith and Science", en Knud Haakonssen (ed.). The Cambridge Companion to Adam Smith. Cambridge: Cambridge University Press, 2006, 112-136; y Schliesser, Eric. "Wonder in the Face of Scientific Revolutions: Adam Smith on Newton's «Proof» of Copernicanism”. British Journal for the History of Philosophy, 13, 2005, 697-732.

18 Labio, Catherine. "Adam Smith's Aesthetics”, en Christopher J. Berry, Maria Pia Paganelli y Craig Smith (eds.). The Oxford Handbook of Adam Smith, 105-125, especialmente 106-108; Griswold, Charles L. "Imagination: Morals, Science, and Arts", en Knud Haakonssen (ed.). The Cambridge Companion to Adam Smith, 22-56, especialmente 46-54; Raphael, D.D. y Skinner, A.S. "General Introduction”, en Adam Smith. Essays on Philosophical Subjects, 1-21, especialmente 15-21; Lindgren, J. Ralph. The Social Philosophy of Adam Smith. La Haya: Martinus Nijhoff, 1973, 3-12; Thomson, Herbert F. “Adam Smith's Philosophy of Science”. The Quaterly Journal of Economics, Vol. 79, No. 2, 1965, 212-233.

19 Jones, Peter. "The Aesthetics of Adam Smith", en Peter Jones y Andrew S. Skinner (eds.). Adam Smith Reviewed. Edimburgo: Edinburgh University Press, 1992, 56-78 (también en Hiroshi Mizuta y Chuhei Sugiyama -eds. Adam Smith: International Perspectives. Londres: Macmillan, 1993, 43-62), a partir del análisis de la biblioteca de Smith, identificó una serie de fuentes contemporáneas como Allan Ramsay, Alexander Gerard, Jean-Baptiste Dubos e, inevitablemente, David Hume. En la nota 5 enumeró una serie de autores, entre los que se hallaban Hutcheson, Diderot y Dubos (a Addison, pese a hallarse en su biblioteca, no lo citó), aunque no consideró relevante a ninguno de estos tres. Longuet-Higgins, H. Christopher. "«The History of Astronomy»", en Peter Jones y Andrew S. Skinner (eds.). Adam Smith Reviewed, 79-92, en pp. 84-85 estableció las conexiones entre la ciencia y el arte en la aproximación de Smith a la astronomía, aunque no incidió en las fuentes posibles de esta visión tan notablemente transgresora. La conexión más clara y equilibrada entre la ciencia, el arte y el uso de la fórmula de la belleza de Hutcheson por parte de Smith (propuesta explícitamente, aunque sin profundizar en ella) se halla en Reisman, D.A. Adam Smith's Sociological Economics. Londres: Croom Helm, 1976,45 y 48. 
Smith. A continuación, expondremos el uso que hizo éste de la imaginación estética y su fundamentación de la misma en la teoría de la visión de Berkeley, evitando el reduccionismo perceptivo de éste. Finalmente, reflexionaremos en la conclusión sobre su concepción multifacética de la ciencia. Aunque incidiremos especialmente en su dimensión cultural y estética porque nos parece menos trabajada que la metodológica, nuestro punto de partida es sintético, pues la relevancia contemporánea de la teoría de la ciencia de Smith reside en su irreductible complejidad.

\section{La Historia de la Astronomía y la fórmula de la belleza}

En HA Smith dejó entre paréntesis el valor de verdad de las distintas teorías astronómicas que se han seguido en el tiempo, importándole más sus dimensiones emocional y social, conectadas con la imaginación. Jonathan Conlin dijo con elegancia que no le interesaba tanto la investigación de la naturaleza como la naturaleza de la investigación. ${ }^{20}$ La conexión sería como sigue:

La filosofía es la ciencia de los principios conectivos de la naturaleza. Tras la máxima experiencia que la observación habitual pueda acumular, en la naturaleza parecen proliferar los hechos solitarios e incoherentes con todo lo que les precede, y que por ende perturban el movimiento cómodo del pensamiento; que hacen que sus ideas se sucedan en saltos y corcovos irregulares, por así decirlo; y que tiende de esta manera a introducir las confusiones y desórdenes ya mencionados. La filosofía, al exponer las cadenas invisibles que conectan todos esos objetos dislocados, pretende traer el orden a este caos de apariencias discordes y chirriantes, apaciguar el tumulto en la imaginación y restaurar en ella, cuando revisa los grandes cambios del universo, el tono de tranquilidad y compostura que le es al tiempo más grato de por sí y más conforme con su naturaleza. La filosofía, en consecuencia, puede ser considerada como una de las artes que se dirigen a la imaginación. ${ }^{21}$

Esta remisión a los principios conectivos partía de Newton, cuyo método fue para Smith el más filosófico, ${ }^{22}$ aunque incidió más en su fuerza conectiva que en su realismo. La importancia concedida a la imaginación muestra también la influencia del Treatise de Hume, ${ }^{23}$ pero la referencia al placer y al displacer hace pensar en fuentes adicionales de naturaleza estética. En este sentido, es relevante su definición de la Filosofía (la Ciencia) como la más sublime de las artes agradables. ${ }^{24} \mathrm{El}$ término "arte" era más amplio para Smith que para nosotros; la ciencia, por ejemplo, en la medida que seguía unos procedimientos que se plasmaban en un producto, era un arte, ${ }^{25}$ aunque Smith precisaba que se dirigía a la imaginación, a la que proporcionaba

\footnotetext{
Conlin, Jonathan. Adam Smith. Londres: Reaktion Books, 2016, 55.

Astronomia, 57; Astronomy, 45-46.

Smith, Adam. Lectures on Rhetoric and Belles Lettres, 145-146.

Raphael, D.D. y Skinner, A.S. "General Introduction", 15-21.

Astronomía, 57; Astronomy, 46.

Labio, Catherine. "Adam Smith's Aesthetics", 112. Posiblemente detrás de esto se halle la entrada "Arte" que Diderot redactó en 1751 para la Enciclopedia. Diderot, Denis. Arte, en Arte, gusto y estética en la Encyclopédie. Valencia: MuVIN, 2005, 23-37, así como D'alembert, Jean le Rond. Discours Préliminaire de l'Encyclopédie (1751). París: Éditions Gonthier, 1965, 52 (en castellano: Discurso preliminar de la Enciclopedia. Traducción de Consuelo Berges. Madrid: Sarpe, 1984, 69).
} 
placer. Le interesaba, por lo tanto, su dimensión estética.

Por eso, creemos necesario buscar fuentes complementarias en la explicación de HA, sin poner en duda el influjo de Newton y Hume. ${ }^{26}$ Addison proporcionó un elemento fundamental, la imaginación y su necesidad de ser gratificada, pero también fueron importantes otros autores que hablaron de la belleza de la ciencia, como Una investigación sobre el origen de nuestras ideas de belleza y virtud de Hutcheson (obra bien conocida por Smith), el Tratado sobre la belleza de Crousaz (del que poseía un ejemplar en su biblioteca ${ }^{27}$ ) o las entradas "Arte" y "Belleza" de Diderot en la Enciclopedia, admirada explícitamente por él. ${ }^{28}$ Cualquiera de ellos podría haber situado a la ciencia entre los placeres de la imaginación, sin dejar de respetar por ello su valor cognoscitivo. ${ }^{29}$

Por seguir un orden cronológico y causal, Crousaz dedicó el Capítulo 9 de su Tratado a la belleza de las ciencias. Por ejemplo, de la belleza de la física dijo:

Si la diversité reduite à l'unité, si l'irrégularité ramenée à l'ordre sont des caracteres réels de Beauté $[\ldots]$ où trouvera-t-on plus de Beauté dans la Physique? Elle vient à bout de ranger exactement cette multitude innombrable de Corps qui composent cet immense Univers dans un petit nombre de genres. ${ }^{30}$

Aunque Crousaz se refería al placer y al asombro que producían las ciencias, al estudiar su función derivó hacia la religión natural, pues consideraba que la unidad que descubrían en un mundo diverso remitía a un Diseñador inteligente. Hutcheson retomó este tema y, pese a ubicarlo también en el discurso de la Teología Natural, lo conectó con la economía mental del sujeto empírico. ${ }^{31}$ En Una investigación se refirió a la belleza de los teoremas, ejemplificándola con la geometría de Euclides y la gravedad de Newton. Por ejemplo, explicó subjetivamente nuestra relación con el teorema 47 de Pitágoras:

Para que podamos advertir del mejor modo posible que este acuerdo, o unidad de una infinitud de objetos en el teorema general, es el fundamento de la belleza o placer que sigue a su descubrimiento, comparemos nuestra satisfacción en tales descubrimientos con el inquieto estado mental en que nos encontramos cuando sólo podemos medir líneas o superficies mediante una escala o cuando hacemos experimentos que no podemos reducir a una regla general, sino sólo apilar en una multiplicidad de observaciones particulares inconexas. Cada una de nuestras tentativas descubre una verdad nueva, pero sin placer ni

26 Incluso se podría plantear que la influencia (estética) de Hume implicaba la influencia de Hutcheson o, mejor, que la influencia de Hutcheson y la de Hume se complementaban desde un punto de vista estético. Eso es lo que deducimos de Ross, Ian. "Aesthetic Philosophy: Hutcheson and Hume to Alison", en Andrew Hook. The History of Scottish Literature, Vol. 2. Aberdeen: Aberdeen University Press, 1987, 239-258, especialmente 240, 243 y $248-250$.

27 Mizuta, Hiroshi. Adam Smith's Library, 16; Yanaihara, Tadao. Catalogue of Adam Smith's Library, 95.

28 Smith, Adam. "A Letter to the Authors of the Edinburgh Review" (1756), en Adam Smith. Essays on Philosophical Subjects, 242-256, especialmente 244-248 (traducción castellana de Carlos Rodríguez Braun. "Una Carta a los Autores de la Edinburgh Review", en Adam Smith. Ensayos filosóficos, 215-218).

29 Labio, Catherine. "Adam Smith's Aesthetics", 106 y 112, menciona algunos de estos temas, aunque por desgracia muy de pasada.

30 Crousaz, Jean-Pierre de. Traité du Beau (1715). París: Fayard, 1985, 175-258 (Capítulo 9), 185 (página citada); también 191 (sobre la física) y 208 (sobre la mecánica).

31 Kivy, Peter. The Seventh Sense. Oxford: Oxford University Press, 2003, 24-62; Dickie, George. El siglo del gusto. Madrid: Antonio Machado Libros, 2003, 21-36. 
belleza, a pesar de la variedad, hasta que podamos descubrir algún tipo de unidad, o las reduzcamos a una regla general. ${ }^{32}$

Este texto anticipaba tanto el marco dentro del cual Smith organizó el argumento central de HA (consistente en que la ciencia progresaba conectando una cantidad creciente de hechos mediante un número decreciente de principios) como la relación del sujeto con dicho progreso. Según Smith la Filosofía, en tanto que ciencia de los principios conectivos de la naturaleza, trataba de reducir la incoherencia y dispersión fenoménica porque éstas "disturb the easy movement of the imagination". ${ }^{33}$ Está clara la conexión con Hutcheson, pues éste también habló de las observaciones incoherentes que generaban un "uneasy state of Mind" 34 que la ciencia podía superar, produciendo satisfacción y placer. Con su HA Smith estaba desarrollando este capítulo de su maestro.

Diderot, admirador de Hutcheson, también desarrolló en la entrada "Belleza" de la Enciclopedia una explicación de la belleza de la ciencia, aunque era menos elegante que la de éste o la de Crousaz. Se basaba en su teoría de la belleza como exposición de relaciones, según la siguiente definición:

La belleza, que consiste siempre en las relaciones, estará en esta ocasión en razón compuesta del número de relaciones y de la dificultad que habría para percibirlas. ${ }^{35}$

Las ciencias mostraban relaciones entre elementos previamente insospechadas, resultando más admirables cuando eran menos obvias y más difíciles de percibir. Con ello resolvían problemas intelectuales y simplificaban la percepción de la realidad, haciéndola más placentera en su nuevo orden descubierto. Las ciencias eran difíciles, pero se acababan simplificando y entonces, en su evidencia, resultaban bellas. Hutcheson lo expresó previamente con más claridad:

Estas son las bellezas que encantan al astrónomo y que tornan placenteros sus aburridos cálculos. ${ }^{36}$

Adam Smith sintonizaba con esto cuando afirmaba que la imaginación, por término medio, era perezosa e indolente, ${ }^{37}$ pues deseaba básicamente no esforzarse. Lo que más la cansaba era el caos disperso y no conectado de la percepción de la realidad, desarrollando teorías para superarlo. Así, afirmó:

32 Hutcheson, Francis. Una investigación sobre el origen de nuestra idea de belleza (1725). Traducción de Jorge V. Arregui. Madrid: Tecnos, 1992, 34 (en inglés: An Inquiry into the Original of Our Ideas of Beauty and Virtue. Indianápolis: Liberty Fund, 2008, 37).

33 Astronomy, 45. Rodríguez Braun traduce "imagination” como "pensamiento" (Astronomía, 57).

34 "Inquieto estado mental" en la traducción proporcionada por Jorge V. Arregui en la anterior cita. Creemos que lo mejor sería traducir "easy" por fácil y "uneasy" por difícil.

35 Diderot, Denis. Investigaciones filosóficas sobre la naturaleza y el origen de lo bello (1751), en Denis Diderot. Pensamientos filosóficos. Investigaciones filosóficas sobre la naturaleza y el origen de lo bello, traducción de Francisco Calvo Serraller. Madrid: Sarpe, 1984, 145. Traducción del Tomo Segundo de la Encyclopédie, entrada "Beau", 179.

36 Hutcheson, Francis. Una investigación..., 27 (An Inquiry..., 31).

37 Astronomía, 93; Astronomy, 86. 
La imaginación, cuando conoce la ley por la cual cualquier movimiento es acelerado o retardado, puede seguirlo y presenciarlo más fácilmente que cuando está desorientada $y$, por así decirlo, vaga en la incertidumbre con relación a la proporción que regula sus variaciones. ${ }^{38}$

Con esto quedaban establecidos los principios básicos que iban a definir la historia narrada en HA. Por mucho que al principio un sistema, como por ejemplo el ptolemaico, haya introducido aquietamiento, la experiencia mostraba constantemente nuevos hechos y la necesidad de integrarlos hacía que los sistemas se fueran volviendo más complejos y difíciles de seguir, generando cada vez más confusión. ${ }^{39}$ Fue esto (y no un experimento crucial que exigiera su sustitución) lo que condujo al sistema copernicano, el cual proporcionaba una trama conectiva más simple de los mismos hechos, siendo más adecuado por ello para la imaginación. ${ }^{40}$ Smith seguía con ello a Diderot, mostrando que la complejidad de las relaciones se hacía más evidente y asumible y, con ello, más bella. Por lo mismo se acabó imponiendo el sistema newtoniano, pues con un único principio, la gravedad, daba cuenta de una cantidad ingente de fenómenos celestes y terrestres, entre los que la imaginación se podía mover de una manera cotidiana. ${ }^{41}$

La historia de las teorías astronómicas era, pues, la historia de las evoluciones de la imaginación que, afrontando el caos perceptivo, construyó sistemas cada vez más coherentes e integradores. Esto nos ha conducido a identificar el tipo de estructura que aquietaba a la imaginación, a saber, aquélla que englobaba la mayor cantidad posible de hechos bajo el menor número de principios. Podríamos decir, con Diderot, que se trataba de armonizar el mayor número posible de relaciones; o, con Crousaz y Hutcheson, que se estaba desarrollado progresivamente la fórmula de la belleza, basada en la razón compuesta de uniformidad (de principios explicativos) más diversidad (de hechos observados). Esto puede resultar más claro si se profundiza un poco en el uso metafórico que hizo Smith de la máquina como expresión máxima de la fórmula de la belleza.

\section{La transversalidad de la máquina}

Es suficientemente conocido que la máquina sirvió como una poderosa metáfora cognoscitiva en los siglos XVII y XVIII. ${ }^{42}$ Smith hizo un importante uso de la misma en HA cuando desarrolló una asombrosa analogía entre los distintos sistemas de pensamiento y las máquinas:

Los sistemas en muchos aspectos se asemejan a las máquinas. Una máquina es un sistema pequeño, creado para desarrollar y para conectar en la realidad los diferentes movimientos y efectos que el artesano ["artist"] necesita. Un sistema es una máquina

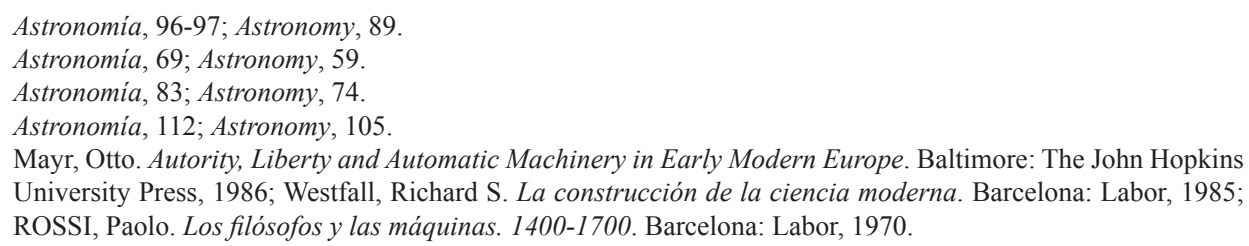


imaginaria inventada para conectar en la mente los diversos movimientos y efectos que ya existen en la realidad. Las máquinas que son primero inventadas para efectuar cualquier marcha concreta son siempre las más complejas, y los artesanos [“artists"] posteriores generalmente descubren que con menos ruedas, con menos principios motrices que los empleados originalmente se pueden producir más fácilmente los mismos efectos. Análogamente, los primeros sistemas son siempre los más complejos y por regla general se cree que es necesario una cadena o principio conectivo para enlazar todos los pares de fenómenos aparentemente desunidos; pero frecuentemente sucede que después se descubre que un solo gran principio conectivo es suficiente para ligar todos los fenómenos desacordes que tienen lugar en el conjunto de una especie de cosas. ${ }^{43}$

Las dos formas de concebir la máquina indicadas en el párrafo citado se correspondían con los sistemas aristotélico y newtoniano, ${ }^{44}$ que quedaban así vinculados con la estética, pues, como veremos, la comparación con la máquina hacía más visible e intuitiva la interpretación de la historia de la ciencia a partir del desarrollo de la fórmula de la belleza. En tanto que objeto construido por el ser humano que evolucionaba minimizando recursos y maximizando efectos, era el artefacto estético por excelencia, el que mejor incorporaba la fórmula de la belleza de Crousaz y Hutcheson, recogida por él en varios lugares. ${ }^{45}$

Crousaz ejemplificó la fórmula con la arquitectura, pero Hutcheson puso numerosos ejemplos mecánicos, lo que condujo a Scott a definir su estética como propia del ingeniero. ${ }^{46}$ Aunque, a diferencia de Smith, Hutcheson usó la máquina para probar la existencia del Dios diseñador, con ella ilustraba la fórmula de la belleza, la cual se podía adaptar sin problemas a un planteamiento más mundano, como quedaba claro en el ejemplo siguiente:

De este modo, ¿quién no aprueba como perfección de un reloj el que tres o cuatro movimientos de las manecillas de la hora, los minutos y los segundos y el disco de los meses sean provocados por sólo un resorte o peso en lugar de por tres o cuatro en una máquina muy compleja que llevara a cabo los mismos efectos y respondiera a los mismos propósitos con igual exactitud? Pues Bien, el fundamento de esta belleza parece claramente ser la uniformidad o unidad de la causa entre la diversidad de los efectos. ${ }^{47}$

Hutcheson propuso, pues, el siguiente criterio para baremar la evolución de las máquinas: había progreso si con una única causa motora (un resorte o un peso) se

43 Astronomía, 75; Astronomy, 66.

44 Smith, Adam. Lectures on Rhetoric and Belles Lettres, 145-146.

45 Por ejemplo, Smith, Adam. The Theory of Moral Sentiments (1759). Indianápolis: Liberty Fund, 1976, 199200; citaremos como TMS (hay traducción castellana de Carlos Rodríguez Braun: Teoría de los sentimientos morales. Madrid: Alianza, 1997, 357; citaremos como TSM); y Smith, Adam. Lectures on Jurisprudence. Indianápolis: Liberty Fund, 1982, 335 y 488 (hay dos ediciones castellanas que recogen sendos manuscritos sobre jurisprudencia reunidos en esta edición: Lecciones sobre Jurisprudencia. Traducción de Manuel Escamilla Castillo y José Joaquín Jiménez Sánchez. Granada: Comares, 1995, 382; y Lecciones de Jurisprudencia. Traducción de Alfonso Ruiz Miguel. Madrid: Centro de Estudios Constitucionales, 1996, 134). Véase Crousaz, Jean-Pierre de. Traité du Beau, 29; y Hutcheson, Francis. Una investigación..., 24 ("lo que llamamos bello en los objetos, para decirlo en términos matemáticos, parece ser una razón compuesta de uniformidad y variedad porque, cuando la uniformidad de los cuerpos es igual, la belleza es equivalente a la variedad y, cuando la variedad es igual, la belleza es equivalente a la uniformidad"; traduce An Inquiry..., 29).

46 Scott, William Robert. Francis Hutcheson. Nueva York: August M. Kelley Publishers, 1966, 191.

47 Hutcheson, Francis. Una investigación, 61; An Inquiry, 58. 
conseguían los mismos efectos (movimientos horarios) para los que previamente hacían falta varias. Era el mismo criterio utilizado por Smith para evaluar el progreso de las ciencias en HA. Además, para Hutcheson no se trataba sólo de algo técnico, sino también estético, pues las máquinas que producían más diversidad a partir de más uniformidad eran más hermosas. Diderot, que conocía estos planteamientos y que en la preparación de las láminas de la Enciclopedia había visitado talleres, montando y desmontando máquinas por sí mismo, sitúo la génesis de los valores estéticos en la experiencia de la máquina, el objeto que mejor ilustraba su definición de la belleza como relación:

[...] toda máquina supone combinación, sincronización de partes tendentes a un mismo fin [...] y pasamos de la noción de una multitud de seres artificiales y naturales, cohesionados, proporcionados, combinados y simétricos, a la noción, positiva y abstracta, de orden, cohesión, proporción, combinación, proporciones y simetría. ${ }^{48}$

Nos parece bastante claro que esto también está detrás de HA, lo que no ha sido considerado aún en este contexto. Como Diderot, ya al comienzo de HA Smith nos lleva a los talleres de los artesanos más comunes para aprender algo de ellos, ${ }^{49}$ como condujo a sus alumnos en sus lecturas sobre jurisprudencia y siguió haciendo hasta La riqueza de las naciones. ${ }^{50} \mathrm{Sin}$ duda, sabía de lo que hablaba cuando se refería a la evolución de las máquinas en el sentido de la fórmula de la belleza y cuando extendía la metáfora hacia otros ámbitos, de lo que dejó numerosos ejemplos en sus escritos. Veamos algunos.

Se trataba de un esquema genérico para analizar el desarrollo de la historia humana, aplicándolo Smith a múltiples procesos, desde la historia del lenguaje hasta las transformaciones de la idea de la divinidad. ${ }^{51}$ También fue usado por él como un modelo a través del cual acceder a la estructura de los fenómenos sociales. En la Teoría de los sentimientos morales la comprensión de la máquina le condujo a la estructuración de la sociedad como un sistema. ${ }^{52}$ Smith utilizó la metáfora en este sentido minuciosamente, lo que le ayudó a clarificar su idea, de origen estoico, del papel social de la virtud. ${ }^{53}$ La conducta virtuosa era útil para la sociedad y nos complacía mediante su similitud con una máquina bien dispuesta, ${ }^{54}$ bien planificada primero, después bien construida y, al final, bien engrasada:

La sociedad humana, cuando la contemplamos desde una perspectiva abstracta y filosófica, parece una gran máquina, una inmensa máquina cuyos movimientos ordenados

48 Diderot, Denis. Investigaciones filosóficas sobre la naturaleza y el origen de lo bello, 125-126 (traducción de Encyclopédie, "Beau”, 175). Sobre la consideración de la máquina véase además D’alembert, Jean le Rond. Discours Préliminaire, 137-141 y 149-150 (en castellano: Discurso preliminar, 147-151 y 160-161).

49 Astronomía, 55; Astronomy, 44.

50 Smith, Adam. Lectures on Jurisprudence, 345-347 y 351 (Lecciones sobre Jurisprudencia, 393-394 y 398-399); WN, I, 19-24 (en castellano RN, 39-43).

51 Smith, Adam. Lectures on Rhetoric and Belles Lettres, 13 y 223; Smith, Adam. The Principles Which Lead and Direct Philosophical Enquiries; Illustrated by the History of the Ancient Physics, en Adam Smith. Essays on Philosophical Subjects, 106-117, especialmente 113-114 (traducción castellana en Ensayos filosóficos, 120121).

52 TSM, 334; TMS, 185.

53 TSM, 508; TMS, 289.

54 TSM, 569; TMS, 326. 
y armoniosos dan lugar a numerosas consecuencias agradables. Con cualquier otra maquinaria hermosa y noble que ha sido producida por el ingenio humano sucede que todo lo que tienda a hacer que sus movimientos sean más suaves y sencillos derivará de ese efecto una cierta belleza y todo lo que tienda a obstruirlos será por tal razón desagradable; del mismo modo la virtud, que por así decirlo es el fino acabado de los engranajes sociales, necesariamente complace, mientras que el vicio, cual herrumbre vil que los hace chirriar y rechinar, es necesariamente ofensivo. ${ }^{55}$

En este pasaje Smith estructuraba los sistemas sociales de la misma manera y con los mismos efectos que los sistemas astronómicos, pues si todo discurría fácilmente ("ease" fue el término común a ambos casos) recibíamos en ambos casos placer $\mathrm{y}$, si no, displacer y ofensa. El tono con el que interpretaba la máquina social era, como resulta evidente en la cita, fuertemente estético, pues la suavidad de la virtud producía un sonido agradable, pero la herrumbre del vicio hacía que la máquina sonara mal, chirriando y rechinando. Esta percepción estética de la sociedad como máquina queda reforzada si atendemos al comienzo de la Teoría de los sentimientos morales, donde situó "la variedad de apariencias en perpetua exhibición por la gran máquina del universo, con los engranajes y resortes secretos que la movilizan" en el mismo ámbito que el paisaje, los ornamentos de un edificio, la composición de un discurso o la expresión de una pintura, identificando "los asuntos generales del saber y el gusto". ${ }^{56}$

El sonido de la máquina como algo estético conduce al análisis que Smith hizo de la música en De la naturaleza de la imitación que tiene en las llamadas artes imitativas, donde definió el ritmo como la regulación de los sonidos formando un sistema. ${ }^{57}$ Cuando concluyó su análisis de la música extendió la idea, reflexionando sobre la estructura de un concierto, una de las expresiones máximas de la fórmula belleza (es decir, de uniformidad más variedad), de la siguiente manera:

Al contemplar esa inmensa multiplicidad de sonidos gratos y melodiosos, arreglados y recopilados, tanto en su coincidencia como en su sucesión, en un sistema completo y regular, la mente en realidad disfruta no sólo de un muy intenso placer sensual sino también de un elevado placer intelectual, no disímil del que deriva de la contemplación de un gran sistema en cualquier otra ciencia. ${ }^{58}$

55 TSM, 552; TMS, 316.

56 TSM, 68; TMS, 19.

57 Smith, Adam. Of the Nature of that Imitation which Takes Place in what are Called the Imitative Arts, en Adam Smith. Essays on Philosophical Subjects, 176-213, citada 187; citaremos como Imitation (traducción castellana De la naturaleza de la imitación..., en Ensayos filosóficos, 173-211, citada 184; citaremos como Imitación). Lógicamente, es el texto en el que se centran casi todos los que estudian la estética de Smith. Véase Labio, Catherine. "Adam Smith's Aesthetics", 109-111; De Marchi, Neil. "Smith on Ingenuity, Pleasure, and the Imitative Arts", en Knud Haakonssen. Tha Cambridge Companion to Adam Smith, 136-157; Malek, James S. "Adam Smith's Contribution to Eighteenth-Century British Aesthetics". The Journal of Aesthetics and Art Criticism, Vol. 3, No. 1, 1972, 49-54; Lindgren, J. Ralph. The Social Philosophy of Adam Smith, 7-12. El texto se contextualiza en la introducción de W.P.D. Wightman a Essays on Philosophical Subjects, 171-175.

58 Imitación, 202 (Imitation, 205). Bryce, J.C. "Introduction", en Adam Smith. Lectures on Rhetoric and Belles Lettres, 36, se esfuerza por remitir este texto a Robert Simpson, su profesor de matemáticas y filosofía natural en Glasgow. No obstante, el planteamiento en términos de arte, belleza y placer remite a una fuente estética, en este caso Hutcheson. 
La conclusión a la que llegamos en este apartado es que entre el sistema solar de Newton, una sociedad bien engrasada a través de la virtud, una máquina moderna y una composición musical no había diferencias estructurales. Además, todos complacían y atraían desinteresadamente a nuestro sentido de la belleza, que respondía a la experiencia de su uniformidad en la diversidad. La metáfora de la máquina en Smith, pues, articulaba el contenido estético que hemos visto surgir en el apartado anterior a partir de fuentes británicas y francesas.

Acabamos de reforzar la importancia de la fórmula de la belleza en HA y, en general, en la obra de Smith. Dando un paso más, en lo que sigue identificaremos su naturaleza visual, cuya conexión con Addison y George Berkeley ayudará a comprender mejor que Smith ponga entre paréntesis el valor de verdad de la ciencia.

\title{
4. La fuerza constructiva de la imaginación
}

Smith situó las teorías científicas entre los placeres de la imaginación, los cuales para Addison eran exclusivamente visuales. ${ }^{59}$ Según éste, un grupo de tales placeres surgían directamente cuando teníamos ante los ojos objetos grandes, singulares o bellos ("great", "uncommon", "beautiful"), lo que estableció los fundamentos de la introducción de HA, que giraba en torno a lo singular ("singular"), lo inesperado ("unexpected") y lo grande y bello ("great or beautiful"). ${ }^{60}$ Lo grande y bello provenía sin modificación de Addison, mientras que los otros dos términos (lo singular y lo inesperado) pueden interpretarse como variaciones de lo inusual. Más que la semántica, sin embargo, lo que nos interesa es la naturaleza visual del proceso, pues tuvo determinadas consecuencias conceptuales.

En HA el planteamiento de Smith fue explícitamente visual y, a partir de ahí, teatral. En todo el texto siguió una retórica muy escenográfica, como cuando se refirió con la siguiente metáfora a la manera en la que la mente humana trabajaba al establecer conexiones entre fenómenos:

\begin{abstract}
¿A quién asombran los mecanismos de un teatro de la ópera después de haber podido ver lo que sucede detrás del escenario? Pero en las maravillas de la naturaleza rara vez podemos descubrir de modo tan transparente dicha cadena de conexión. En verdad, sólo en muy pocas hemos sido admitidos detrás del escenario como para que nuestro asombro finalice totalmente. ${ }^{61}$
\end{abstract}

Podemos desarrollar la analogía ya expuesta entre una teoría científica y una composición musical llevando las teorías a la escena, viendo el decorado que las enmarca y la maquinaria que las controla. Smith creía que nos enfrentábamos visualmente al mundo y que queríamos recrear lo mejor que podíamos la maquinaria

59 Addison, Joseph. "Pleasures of imagination" (1712), en The Spectator, Vol. III. Londres: J.M. Dent and Sons, 1945, 277 (hay edición castellana: Los placeres de la imaginación. Madrid: Visor, 1991, 130-131).

60 Addison, Joseph. "Pleasures of imagination", 279; Adam Smith. Lectures on Rhetoric and Belles Lettres, 64; Astronomía, 43; Astronomy, 33. Labio, Catherine. "Adam Smith's Aesthetics", 106-107 repara en esta conexión. Raphael, D.D. Adam Smith. Oxford: Oxford University Press, 1985, 108, deriva esto a partir de Platón. Sin embargo, para Lázaro Cantero, Raquel. La sociedad comercial en Adam Smith, 113, esto es original de Smith. Lo original de Smith sería, en realidad, el contexto en el que los usó.

61 Astronomía, 54; Astronomy, 42-43. 
que le subyacía. Pudo adoptar una perspectiva teatral porque situó al hecho científico entre los placeres de la imaginación, es decir, porque le interesó ante todo como un fenómeno cultural. Así es como se debe interpretar su programa de investigación:

Examinemos, entonces, los diferentes sistemas de la naturaleza que en estas partes occidentales del mundo, las únicas partes donde sabemos algo de su historia, han sido adoptados sucesivamente por las personas sabias e ingeniosas; $\mathrm{y}$, sin considerar su absurdo o verosimilitud, su acuerdo o incompatibilidad con la verdad y la realidad, estudiémoslos sólo desde el enfoque particular que corresponde a nuestro tema, y limitémonos a investigar el grado en que cada uno de ellos estaba preparado para aliviar la imaginación, para transformar el teatro del mundo en un espectáculo más coherente y por ello más magnífico de lo que podría haber parecido en otro caso. ${ }^{62}$

La expresión "teatro de la naturaleza" podía ser una fórmula retórica heredada del siglo XVII, cuando se usó con profusión, pero en HA la teatralización de la ciencia fue tan recurrente que nos aconseja ir más allá de la metáfora. Para poder hacerlo nos apoyaremos en dos textos redactados en épocas diversas, aunque publicados conjuntamente con HA en los Ensayos filosóficos, a saber, De los sentidos externos e Imitación..., éste citado en el anterior apartado.

La última obra permite fijar algo más el marco estético de HA. En ella Smith elaboró un concepto de imitación artística que se basaba en el rechazo de la copia literal de la realidad, haciendo residir todo el valor en la falta de identidad física y estructural entre la copia y el modelo. ${ }^{63}$ Así, si una pintura copiaba a otra no era imitación sino plagio sin valor, dada la identidad de la naturaleza material de ambas, pero si imitaba a una escena natural sí era algo meritorio y valioso. Su idea era que el placer que sentíamos ante una imitación estaba en relación directa con la diferencia de naturaleza material entre la copia y el original; en el caso de la pintura, entre las tres dimensiones del mundo y las dos dimensiones del cuadro. ${ }^{64} \mathrm{El}$ valor del arte consistía en superar dicha disparidad, por lo que más que de imitación habría que hablar de traducción de un medio a otro. ${ }^{65}$ Por otra parte, al traducir al modelo la copia nos permitía conocerlo en parte; no era el modelo ni jamás lo sería, pero revelaba algunas características suyas.

En HA Smith usó la metáfora teatral para referirse a los sistemas astronómicos, lo que los convertía en una suerte de imitación que traducía al "lenguaje" de nuestros ojos una realidad que no era visual. Así caracterizó, por ejemplo, al sistema ptolemaico:

[...] y allí contemplaron la más hermosa y magnífica parte del gran teatro de la naturaleza, dispuesta y construida de manera tal que podían seguir con comodidad y deleite todas las evoluciones y cambios que en ella ocurrían. ${ }^{66}$

62 Astronomía, 57; Astronomy, 46.

63 Imitación, 175; Imitation, 178.

64 Imitación, 176; Imitation, 179.

65 Disentimos de Labio, Catherine. “Adam Smith's Aesthetics”, 110, donde establece que, a diferencia del filósofo natural, que trata de superarla, el artista realza tal disparidad. Creemos que ésta es, para el arte imitativa, un punto de partida a superar. La excelencia del artista, según Smith, reside en la superación de la disparidad sin la anulación de las diferencias estructurales entre la imitación y lo imitado. En ese sentido, no se diferenciaría radicalmente de la ciencia.

66 Astronomía, 72; Astronomy, 62. 
Es como si aquellos antiguos hubieran estado sentados en el teatro asistiendo a la representación visual del sistema planetario, una representación bidimensional de un universo profundo que se les escapaba.$^{67}$ Esto nos conduce a las consideraciones de Smith sobre la visión en De los sentidos externos, básicamente a su admiración por Berkeley, cuya obra sobre el tema consideró definitiva. ${ }^{68}$

Berkeley mostró que la visión por sí misma no proporcionaba conocimiento de la profundidad, requiriéndose para ello la asociación con la experiencia táctil, la única que nos transmitía la tridimensionalidad. ${ }^{69}$ A partir de aquí Smith definió el espacio visual como algo plano, ajeno a la tercera dimensión:

El mundo tangible, así como todas las diferentes partes que lo componen, tiene tres dimensiones: largo, ancho y profundidad. El mundo visible, y todas las diferentes partes que lo componen, tiene sólo dos: largo y ancho. Se nos presenta sólo como un plano o superficie que, por ciertas sombras y combinaciones de colores, nos sugiere y representa (como lo hace un cuadro) ciertos objetos tangibles que carecen de color, y que por tanto no se parecen en nada a esas sombras y combinaciones de colores. ${ }^{70}$

La experiencia visual era pictórica y a través de la imaginación, que la asociaba con el tacto, traducía un mundo tridimensional en algo diferente a él. ${ }^{71} \mathrm{Si}$ era así en el mundo de los objetos cotidianos que tocamos con nuestras manos, lo era mucho más con respecto a las distancias cósmicas, cuyos límites fueron suprimidos por Descartes,${ }^{72}$ resultándonos imposible tocar su tridimensionalidad. ${ }^{73}$ Puesto que nuestra experiencia de los astros era visual, nunca pasábamos del proscenio, lo que no negaba que la ciencia progresara.

La aceptación de la teoría de Berkeley tenía otras consecuencias epistémicas de más envergadura. No pensamos en la famosa reducción del "esse" al "percipi", en la negación de que las cosas tuvieran existencia más allá de la mente que las percibe,$^{74}$ sino en la definición de la visión como una suerte de lenguaje que Dios nos concedió. ${ }^{75}$ No nos interesa el argumento teológico, que Smith, por otra parte, no negó, sino el lingüístico:

67 Cuando en la "Lecture XXI" de sus Lectures on Rhetoric Adam Smith se refirió a las unidades de interés, tiempo y espacio en el teatro, lo justificó recurriendo a las mismas necesidades conectivas de la imaginación que daban lugar a las teorías científicas, es decir, a su libre movimiento por una trama conectiva cuyos eslabones estaban todos presentes, incidiendo en que eso era lo que nos importaba y producía placer, no el valor de verdad de lo representado en la escena. Smith, Adam. Lectures on Rhetoric, 117-127.

68 Smith, Adam. De los sentidos externos, en Adam Smith. Ensayos filosóficos, 137-171, especialmente 150151; citaremos como Sentidos externos (original inglés Of the External Senses, en Essays on Philosophical Subjects, 135-168, 148; citaremos como External Senses). Sobre la conexión de este ensayo con HA desde una perspectiva estética, véase Brown, Maurice. Adam Smith 's Economics. Londres: Croom Helm, 1988, 25-50.

69 Berkeley, George. Ensayo de una nueva teoría de la visión (1709). Buenos Aires: Aguilar, 1973, $52-53$ (en inglés An Essay towards a New Theory of Vision, en George Berkeley. Philosophical Works. Londres: J.M. Dent \& Sons, 1989, 20-21). Véase Atherton, Margaret. Berkeley's Revolution in Vision. Ithaca: Cornell University Press, 1990, 61-88.

$70 \quad$ Sentidos externos, 153; External Senses, 150-151.

71 TSM, 258; TMS, 135.

72 Astronomía, 101; Astronomy, 94.

73 Sentidos externos, 154; External Senses, 151.

74 Berkeley, George. Tratado sobre los principios del conocimiento humano (1710), en George Berkeley. Tratado sobre los principios del conocimiento humano. Alcifrón. Traducción de Carlos Mellizo. Madrid. Gredos, 2015, 26 (en inglés: A Treatise Concerning the Principles of Human Knowledge, en Philosophical Works, 78).

75 Jantzen, Benjamin C.: An Introduction to Design Arguments, 78-90. 
Los objetos de la vista, como subraya acertadamente el Dr. Berkeley, constituyen una suerte de lenguaje que el autor de la naturaleza dirige a nuestros ojos y por medio del cual nos informa acerca de muchas cosas, cuyo conocimiento resulta para nosotros de la máxima importancia. Así como en el lenguaje corriente las palabras o sonidos no guardan semejanza alguna con las cosas que denotan, en este otro lenguaje los objetos visibles no guardan similitud alguna con el objeto tangible que representan. ${ }^{76}$

Para Adam Smith el referente del lenguaje era importante. ${ }^{77}$ Si Berkeley hablaba de las cosas "significadas" ("signified"), Smith lo hacía de las cosas "denotadas" ("denoted"), lo que daba más importancia al referente extra-lingüístico. ${ }^{78}$ Con todo, aceptaba la diferencia estructural entre la imagen y el objeto, de manera que la fidelidad total de la una al otro no estaba garantizada. Berkeley creía que Dios nos concedió un instrumento fiable, pero Smith declinó recurrir a este deus ex machina que desde la época de Descartes servía para superar el escepticismo radical del nuevo sujeto. ${ }^{79}$

Llegados a este punto, para comprender la propuesta de Smith en HA vamos a retornar a la metodología newtoniana de análisis y síntesis, con la que abríamos este artículo. Para Smith la historia de la ciencia, entre los extremos de la dispersión inicial en la época pre-filosófica y la unificación final en el sistema newtoniano, era en parte un proceso subjetivo mediante el que se desarrollaba la fórmula de la belleza. Era algo estético que surgía de nuestra relación visual con el mundo, al que accedíamos, a través de una suerte de imitación pictórica, con unos medios y procedimientos que no concordaban con la realidad. Para superar el escepticismo que se podía seguir de esto, Smith adoptó (y adaptó) el método Newtoniano de aproximación progresiva a la realidad. Por lo tanto, armonizó una metodología de investigación experimental, proveniente de Newton, con la fórmula de la belleza de Crousaz y Hutcheson, dando lugar esta síntesis a un proceso progresivo de confluencia creciente entre un conocimiento de la realidad cada vez más fiable y la construcción de una imagen del universo cada vez más hermosa.

\section{Conclusiones}

En la interpretación y valoración históricas de la obra de Smith se observan tres tendencias generales que siguen vigentes, aunque están comenzando a revisarse. En primer lugar, se lo sigue leyendo ante todo como el autor de La riqueza de las naciones o (de una manera menos popular) de La teoría de los sentimientos morales, aunque hoy día se acepta que ambas obras se publicaron secuencialmente como parte de un plan global inconcluso. ${ }^{80}$

76 Sentidos externos, 159; External Senses, 156.

77 Véase Glenney, Bryan. "Adam Smith and the Problem of the External World". The Journal of Scottish Philosophy, 9, 2, 2011, 205-223.

78 Berkeley, George. Ensayo, 119 (An Essay, 52); Smith, Adam. Sentidos externos, 159; External Senses, 156.

79 Sobre este complejo tema véase Fernández Rodríguez, José Luis. El Dios de los filósofos modernos: de Descartes a Hume. Pamplona: EUNSA, 2008.

80 Es el punto de partida crítico de la popular obra de Roberts, Russell. Cómo Adam Smith puede cambiar tu vida. Barcelona: Antoni Bosch, 2016. Sobre su "gran proyecto" integrador véase Phillipson, Nicholas. "Adam Smith: A Biographer's Reflections", en Christopher J. Berry, Maria Pia Paganelli y Craig Smith (eds.). The Oxford Handbook of Adam Smith, 23-35. 
En segundo lugar, la posición del intérprete suele afectar a la interpretación más que en otros casos. Smith es diferente para un historiador de la Economía y para un historiador de la Filosofía, lo que se agudiza si introducimos problemas ideológicos, como la medida de su liberalismo o la intensidad de su escepticismo. Con su obra se podría justificar casi cualquier cosa porque fue un filósofo transversal, lo que exige una lectura integral de la misma, que profundice en escritos aparentemente menos importantes, como HA. ${ }^{81}$

En tercer lugar, hay factores estructurales que se aplican más específicamente a HA. Pensamos en el prestigio de la ciencia, valorada como algo ajeno a la estética, de manera que a partir de cierto punto la presencia de ésta parece una distorsión de aquélla, lo que hace que los intérpretes de HA planteen la presencia de la ciencia y de la estética como una contradicción que exige la elección de una u otra. Puesto que en el siglo XVIII esa dicotomía no existía, lo mejor es la aproximación integral al texto. Nosotros hemos incidido en la dimensión estética porque considerábamos que había fuentes no detectadas respecto a ella, no porque sea más importante o porque excluya la ciencia.

Dejando en suspenso (no negando) el valor de verdad de la ciencia, Smith la definió como algo a la vez histórico, metodológico, social y personal. Su proyecto trató de integrar todas estas dimensiones, lo que produjo una complejidad que se podría interpretar como tensión y dicotomía, con notables consecuencias sobre la interpretación de su teoría del conocimiento. Nosotros, en nuestra conclusión, nos alineamos con Warren J. Samuels y Jerry Evensky, aunque, a diferencia de ellos, que interpretaban una conexión tensa entre ambas dimensiones, vemos una conexión de reforzamiento mutuo. ${ }^{82}$

Smith pensaba que el orden que las teorías generaban lo instaurábamos en parte nosotros mismos a lo largo de la historia, lo que explica la complejidad de su propuesta, que se alimentó a la vez de la estética de Hutcheson (que explicaba la relación emocional del sujeto con la teoría) y de la metodología de Newton (que presentaba el proceso como una aproximación histórica a la realidad). Eso se aplicaba tanto a las teorías establecidas como a su puesta en duda y quiebra, pues las nuevas teorías no sólo se originaban ante nuevos hechos no asumibles por las anteriores, sino también gracias al trabajo de determinados sujetos (los científicos creadores) cuya imaginación, amando a la vez la novedad y el orden, generaba, pasado el tiempo, una nueva coyuntura armonizada. Smith detectó, pues, dos dimensiones que interactuaban, una estética y otra epistémica. El deseo de orden de la imaginación, en tanto se manifestaba como displacer, era estético, pero dinamizaba una búsqueda de naturaleza epistémica cuyos logros, al producir placer, volvían a ser estéticos. Se trataba de un proceso recurrente cuyo final no se contemplaba.

De una lectura no excluyente de HA surge una visión smithiana de la ciencia que no era relativista ni limitada. La existencia de un aspecto estético en la dinámica de

81 Véase Haakonssen, Knud y Winch, Donald. "The Legacy of Adam Smith”, en Knud Haakonssen (ed.). The Cambridge Companion to Adam Smith, 366-394, especialmente 372. Como modelo de aproximación integral proponemos Labio, Catherine. "Adam Smith's Aesthetic". Sobre cómo se puede justificar casi todo a partir de retazos de la obra de Smith, remitimos a la quinta parte de Hanley, Ryan Patrick (ed.). Adam Smith. His Life, Thought, and Legacy. V. Smith Beyond the Academy, 461-557.

82 Samuels, Warren J. Erasing the Invisible Hand. Cambridge: Cambridge University Press, 2014, 83-108, especialmente 86-87; Evensky, Jerry. Adam Smith's Moral Philosophy. Nueva York: Cambridge University Press, 2005, 3-33; también Otteson, James R. Adam Smith. Londres: Bloomsbury Academy, 2013, 27-30. 
las teorías no negaba que Newton superase a Descartes y éste a Ptolomeo, algo tan evidente como que las máquinas que estaban permitiendo la Revolución Industrial eran mejores que las helenistas. Smith, que era un progresista ilustrado, no lo dudaba. Era un hecho, por otra parte, que no había límites en este desarrollo, que la ciencia siempre iría más allá de sí misma en el conocimiento de la realidad (es decir, tarde o temprano Newton sería superado), aunque sin cancelar su naturaleza aproximativa.

Por otro lado, dicho progreso también era una realización cada vez más lograda de la fórmula de la belleza, una composición cada vez más placentera de uniformidad más variedad. Este desarrollo era uno y el mismo que el del conocimiento del mundo, pues la fórmula daba lugar a que los principios explicativos fueran cada vez menos y sus consecuencias, sin embargo, más numerosas. Este criterio de belleza generaba sofisticación epistémica $\mathrm{y}$, con ella, un mayor grado de aproximación a la realidad, pues los nuevos principios, más integradores, incluían fenómenos que previamente no se habían observado. Con ello el paisaje de hechos a los que se accedía no dejaba de crecer, asegurando cada vez más la corrección de la teoría. Si los nuevos datos eran inasumibles, es decir, si se producía una merma en uniformidad, surgía la necesidad de reformular los principios o crear unos nuevos que acabaran integrando la dispersión surgida del anterior sistema con los nuevos hechos. Ese era el proceso histórico de la ciencia, un progreso objetivo y medible que no dependía de nuestro capricho, aunque adoptara la fórmula de la belleza de Hutcheson y Crousaz. En el desarrollo de dicha fórmula coincidían, pues, el aumento de nuestro conocimiento de la realidad y nuestra capacidad de gozar de la misma. Gracias a la ciencia, el asombro daba paso progresivamente a la belleza, con lo cual Smith mostraba que los aspectos metodológicos, epistemológicos y culturales de la ciencia eran inescindibles, parte de una misma realidad humana.

Podemos considerar esta propuesta como una de las manifestaciones más potentes del Neoclasicismo en ciencia. ${ }^{83}$ Hay método científico porque hay orden en la diversidad, es decir, porque hay belleza, la cual, a su vez, es la manifestación más placentera del método. Smith escribía esto antes de la escisión cultural entre las ciencias y las humanidades que tuvo lugar definitivamente en la segunda mitad del siglo XIX. La suya era una época aún no escindida, lo que se mostraba plenamente en HA. Por eso mismo, para nosotros representa una alternativa a nuestra cultura, escindida entre las ciencias y las humanidades. Smith nos muestra que la ciencia llevada al extremo se vuelve arte, sin dejar de ser por ello una búsqueda racional de conexiones que expliquen todo lo explicable con la mayor economía posible de medios, pero también que el arte llevado a su extremo se vuelve ciencia, la máxima conexión de la unidad de los principios con la multiplicidad de los resultados, sin dejar por ello de causarnos una profunda complacencia.

Parte del atractivo de la visión de Smith para una época como la nuestra es que no recurre a la hipótesis de Dios, como hizo Newton, pero tampoco al determinismo metodológico de Laplace. ${ }^{84}$ Newton afirmó en uno de sus manuscritos teológicos:

\footnotetext{
83 Usamos el término "Neoclasicismo" tal y como está establecido en Historia del Arte, a saber, como el estilo propio de la segunda mitad del siglo XVIII y comienzos del XIX.

84 Laplace, Pierre-Simon de. Ensayo filosófico sobre las probabilidades (1814). Madrid: Alianza, 1985, 25; Weinert, Friedel. The Demons of Science. Berlin, Springer, 2016, 65-73.
} 
Truth is ever to be found in simplicity, and not in the multiplicity and confusion of things. As the world, which to the naked eye exhibits the greatest variety of objects, appears very simple in its internal constitution when surveyed by a philosophic understanding, and so much the simpler by how much the better it is understood, so it is in these visions. It is the perfection < all $>$ God's Works that they are done with the greatest simplicity. He is the God of order and not of confusion. ${ }^{85}$

Smith pensaba, al igual que Hutcheson o Crousaz, en términos muy similares, si bien desplazó a Dios y puso al ser humano en su lugar. La conjunción de multiplicidad y simplicidad no era para él algo garantizado por Dios sino la proyección de un sujeto empírico empujado por sus propias emociones. Desde una posición radicalmente mundana, pensaba que con eso nos íbamos acercando a la verdad y, al mismo tiempo, realizando la belleza. Si eso no nos resulta convincente es que seguimos viviendo en una cultura escindida, cultura que, pese a nuestros esfuerzos, no hemos superado.

\section{Referencias bibliográficas}

Atherton, Margaret. Berkeley's Revolution in Vision. Ithaca: Cornell University Press, 1990. Berkeley, George. Ensayo de una nueva teoría de la visión. Buenos Aires: Aguilar, 1973.

Berkeley, George. Philosophical Works. Londres: J.M. Dent \& Sons, 1989.

Berkeley, George. Tratado sobre los principios del conocimiento humano. Alcifrón. Madrid. Gredos, 2015.

Berry, Christopher J., Maria Pia Paganelli y Craig Smith (eds.). The Oxford Handbook of Adam Smith. Oxford: Oxford University Press, 2016.

Bonar, James. A Catalogue of the Library of Adam Smith. Londres: Macmillan and Co., 1932.

Bréban, Laurie. "Smith oh Happiness: Towards a Gravitational Theory". European Journal of History of Economic Thought, Vol. 21, No. 3, 2014, 359-391.

Brown, Maurice. Adam Smith's Economics. Londres: Croom Helm, 1988.

Campbell, R.H. y Andrew S. Skinner (eds.). The Origins and Nature of the Scottish Enlightenment. Edimburgo: John Donald Publishers, 1982.

Conlin, Jonathan. Adam Smith. Londres: Reaktion Books, 2016.

Cremaschi, Sergio. "Adam Smith: Skeptical Newtonianism, Disenchanted Republicanism, and the Birth of Social Science", en Marcelo Dascal y Ora Gruengard (eds.). Knowledge and Politics. Boulder: Westview Press, 1989, 83-110.

Cremaschi, Sergio. "La Herencia Newtoniana en la Economía Política del Siglo XVIII", en Alberto Elena, Javier Ordóñez y Mariano Colubi (eds.). Después de Newton: ciencia y sociedad durante la primera Revolución Industrial. Barcelona: Anthropos, 1998, 70-101.

Crousaz, Jean-Pierre de. Traité du Beau. París: Fayard, 1985.

D'alembert, Jean le Rond. Discours Préliminaire de l'Encyclopédie. París: Éditions Gonthier, 1965.

D’alembert, Jean le Rond. Discurso preliminar de la Enciclopedia. Madrid: Sarpe, 1984.

Dickie, George. El siglo del gusto. Madrid: Antonio Machado Libros, 2003.

Diderot, Denis. Arte, en Arte, gusto y estética en la Encyclopédie. Valencia: MuVIN, 2005, 23-37.

Diderot, Denis. Pensamientos filosóficos. Investigaciones filosóficas sobre la naturaleza y el

85 Newton, I. "Fragments from a Treatise on Revelation", en Manuel, Frank E. The Religion of Isaac Newton. Oxford: Clarendon Press, 1974, 120. 
origen de lo bello. Madrid: Sarpe, 1984.

Diemer, Arnaud y Guillemin, Hervé. 'L’Économie Politique au Miroir de la Physique: Adam Smith et Isaac Newton". Revue d'Histoire des Sciences, 64, 2011, 5-26.

Evensky, Jerry. Adam Smith's Moral Philosophy". Nueva York: Cambridge University Press, 2005.

Fernández Rodríguez, José Luis. El Dios de los filósofos modernos: de Descartes a Hume. Pamplona: EUNSA, 2008.

Fiori, Stefano. "Adam Smith on Method: Newtonianism, History, Institutions, and the «Invisible Hand»". Journal of the History of Economic Thought, Vol. 34, 3, 2012, 411 435.

Glenney, Bryan. "Adam Smith and the Problem of the External World". The Journal of Scottish Philosophy, 9, 2, 2011, 205-223.

Haakonssen, Knud (ed.). The Cambridge Companion to Adam Smith. Cambridge: Cambridge University Press, 2006.

Hanley, Ryan Patrick (ed.). Adam Smith. His Life, Thought, and Legacy. Princeton: Princeton University Press, 2016.

Herman, Arthur. The Scottish Enlightenment. Londres: Fourth State, 2003.

Hetherington, Norriss S. "Isaac Newton's Influence on Adam Smith's Natural Laws in Economics". Journal of the History of Ideas, Vol. 44, No. 3, 1983, 497-505.

Hook, Andrew. The History of Scottish Literature, Vol. 2. Aberdeen: Aberdeen University Press, 1987.

Hume, David. A Treatise of Human Nature. Oxford: Clarendon Press, 1946.

Hume, David. Tratado de la naturaleza humana. Madrid: Tecnos, 1988.

Hutcheson, Francis. An Inquiry into the Original of Our Ideas of Beauty and Virtue. Indianápolis: Liberty Fund, 2008.

Hutcheson, Francis. Una investigación sobre el origen de nuestra idea de belleza. Madrid: Tecnos, 1992.

Jantzen, Benjamin C. An Introduction to Design Arguments. Cambridge: Cambridge University Press, 2014.

Jones, Peter y Skinner, Andrew S. Adam Smith Reviewed. Edimburgo: Edinburgh University Press, 1992.

Kennedy, Gavin. “Adam Smith's Use of the «Gravitation» Metaphor”. Economic Thought, 4, 1, 2015, 67-79.

Kivy, Peter. The Seventh Sense. Oxford: Oxford University Press, 2003.

Laplace, Pierre-Simon de. Ensayo filosófico sobre las probabilidades. Madrid: Alianza, 1985.

Lázaro Cantero, Raquel. La sociedad comercial en Adam Smith. Método, moral, religión. Pamplona: EUNSA, 2002.

Lindgren, J. Ralph. The Social Philosophy of Adam Smith. La Haya: Martinus Nijhoff, 1973.

Malek, James S. “Adam Smith's Contribution to Eighteenth-Century British Aesthetics". The Journal of Aesthetics and Art Criticism, Vol. 3, No. 1, 1972, 49-54.

Manuel, Frank E. The Religion of Isaac Newton. Oxford: Clarendon Press, 1974.

Mayr, Otto. Autority, Liberty and Automatic Machinery in Early Modern Europe. Baltimore: The John Hopkins University Press, 1986.

Mizuta, Hiroshi. Adam Smith's Library. A Catalogue. Oxford: Oxford University Press, 2000.

Mizuta, Hiroshi y Sugiyama, Chuhei. Adam Smith: International Perspectives. Londres: Macmillan, 1993.

Montes Lira, Leónidas. "La Influencia de Newton en Adam Smith”. Anuario Filosófico, XLII, 1, 2009, 137-158.

Newton, Isaac. Four Letters from Sir Isaac Newton to Doctor Bentley (1756), en I. Bernard 
Cohen y Robert E. Schofield (eds.). Isaac Newton's Papers \& Letters on Natural Philosophy. Cambridge/Mass.: Harvard University Press, 1978.

Newton, Isaac. Óptica. Madrid: Alfaguara, 1997.

Newton, Isaac. Opticks. Nueva York: Prometheus Books, 2003.

Newton, Isaac. Principios matemáticos de la filosofía natural. Madrid: Tecnos, 1987.

Otteson, James R. Adam Smith. Londres: Bloomsbury Academy, 2013.

Raphael, D.D. Adam Smith. Oxford: Oxford University Press, 1985.

Raphael, D.D. "«The true old Humean philosophy» and its influence on Adam Smith", en G.P. Morice (ed.). David Hume. Bicentenary Papers. Edimburgo: Edinburgh University Press, 1977, 23-38.

Reisman, D.A. Adam Smith's Sociological Economics. Londres: Croom Helm, 1976.

Roberts, Russell. Cómo Adam Smith puede cambiar tu vida. Barcelona: Antoni Bosch, 2016.

Ross, Ian Simpson. The Life of Adam Smith. Oxford: Clarendon Press, 1995.

Rossi, Paolo. Los filósofos y las máquinas. 1400-1700. Barcelona: Labor, 1970.

Samuels, Warren J. Erasing the Invisible Hand. Cambridge: Cambridge University Press, 2014.

Schliesser, Eric. "Copernican Revolutions Revisited in Adam Smith by Way of David Hume". Revista Empresa y Humanismo, Vol. XIII, 1, 2010, 213-248.

Schliesser, Eric. "Wonder in the Face of Scientific Revolutions: Adam Smith on Newton's «Proof» of Copernicanism". British Journal for the History of Philosophy, 13, 2005, 697-732.

Scott, W.R. Adam Smith as Student and Professor. Glasgow: Jackson, Son \& Company, 1937.

Scott, W. R. Francis Hutcheson. Nueva York: August M. Kelley Publishers, 1966.

Smith, Adam. An Inquiry into the Nature and Causes of the Wealth of Nations. Indianápolis: Liberty Fund, 1981.

Smith, Adam. Ensayos filosóficos. Madrid: Pirámide, 1998.

Smith, Adam. Essays on Philosophical Subjects. Indianápolis: Liberty Fund, 1992.

Smith, Adam. La riqueza de las naciones. Madrid: Alianza, 1994.

Smith, Adam. Lecciones de Jurisprudencia. Madrid: Centro de Estudios Constitucionales, 1996.

Smith, Adam. Lecciones sobre Jurisprudencia. Granada: Comares, 1995.

Smith, Adam. Lectures on Jurisprudence. Indianápolis: Liberty Fund, 1982.

Smith, Adam. Lectures on Rhetoric and Belles Lettres. Indianápolis: Liberty Fund, 1985.

Smith, Adam. Teoría de los sentimientos morales. Madrid: Alianza, 1997.

Smith, Adam. The Theory of Moral Sentiments. Indianápolis: Liberty Fund, 1976.

The Spectator, Vol. III. Londres: J.M. Dent and Sons, 1945.

Thomson, Herbert F. “Adam Smith's Philosophy of Science”. The Quaterly Journal of Economics, Vol. 79, No. 2, 1965, 212-233.

Townsend, Dabney. "Lockean Aesthetics". The Journal of Aesthetics and Art Criticism, Vol. 49, No. 4, 1991, 349-361.

Weinert, Friedel. The Demons of Science. Berlin, Springer, 2016.

Westfall, Richard S. La construcción de la ciencia moderna. Barcelona: Labor, 1985.

Yanaihara, Tadao. Catalogue of Adam Smith's Library in the Possession of the University of Tokyo. Nueva York: Augustus M. Kelley, 1966. 